\title{
NUEVAS COMBINACIONES EN MONOCOTILEDÓNEAS MEXICANAS III (ORCHIDACEAE, POACEAE)
}

\author{
Ana Rosa López-Ferrari \\ Adolfo Espejo Serna \\ Herbario Metropolitano \\ Departamento de Biología, C.B.S. \\ Universidad Autónoma Metropolitana \\ Unidad Iztapalapa \\ Apartado postal 55-535 \\ 09340 México, D.F. \\ e-mail: aes@xanum.uam.mx
}

\section{RESUMEN}

Se proponen las siguientes nuevas combinaciones: Anthaenantia villaregalis (McVaugh \& $R$. Guzmán) Espejo \& López-Ferrari, Panicum macrospermum (Gould) Espejo \& López-Ferrari, Urochloa jaliscana (F. Santana) Espejo \& López-Ferrari, Prosthechea pastoris (Lex.) Espejo \& López-Ferrari e Isochilus bracteatus (Lex.) Salazar \& Soto Arenas y se designan los neotipos de Epidendrum pastoris Lex. y de Dendrobium bracteatum Lex.

\section{ABSTRACT}

The following new combinations are provided: Anthaenantia villaregalis (McVaugh \& R. Guzmán) Espejo \& López-Ferrari, Panicum macrospermum (Gould) Espejo \& López-Ferrari, Urochloa jaliscana (F. Santana) Espejo \& López-Ferrari, Prosthechea pastoris (Lex.) Espejo \& López-Ferrari and Isochilus bracteatus (Lex.) Salazar \& Soto Arenas. The neotypes of Epidendrum pastoris Lex. and Dendrobium bracteatum Lex. are designated.

A partir de 1993 iniciamos la publicación de "Las Monocotiledóneas Mexicanas, una Sinopsis Florística. 1. Lista de Referencia", obra en la cual reunimos los nombres de todas las especies de dicho grupo que crecen en el país, indicando tanto su sinonimia nomenclatural como taxonómica, así como las citas bibliográficas originales, los datos del material tipo y la distribución de los taxa a nivel estatal. Hasta el momento se han publicado ocho fascículos que incluyen la información referente a 45 familias.

Durante el desarrollo del mencionado trabajo nos hemos enfrentado a diversos problemas tanto de índole taxonómica como nomenclatural, entre los cuales se encuentra el constante cambio en la delimitación genérica de algunos grupos. Un ejemplo de esto es el caso de ciertos géneros de gramíneas como Bouteloua, Buchlomimus, Buchloë, Cathestecum, Opizia, Panicum, Dichanthelium, Stipa, Nasella, Urochloa y Brachiaria, entre otros. 
Debido a lo anterior y con el fin de validar algunos nombres para la publicación del fascículo final del listado de referencia en cuestión en el que aparecerán, entre otras, las gramíneas, son necesarias las combinaciones nomenclaturales que a continuación proponemos:

\section{POACEAE}

Anthaenantia villaregalis (McVaugh \& R. Guzmán) Espejo \& López-Ferrari, comb. nov. Basónimo: Leptocoryphium villaregalis McVaugh \& R. Guzmán, in McVaugh, Fl. NovoGaliciana 14: 218-220, f. 15. 1983. TIPO: Jalisco, mpio. Talpa, entre Cuale y La Cumbre Blanca, cañada, 1305 m, 4.IX.1971, R. González Tamayo 369 (holotipo: MICH!; foto UAMIZ 1671!).

Distribución: JAL.

Panicum macrospermum (Gould) Espejo \& López-Ferrari, comb. nov. Basónimo: Dichanthelium macrospermum Gould, Brittonia 32: 358. 1980, non P. macrospermum Spreng., 1825. TIPO: San Luis Potosí, 30 miles E San Luís Potosí, in moist, oak woodland at 2300 m, 13.VII.1963, McGregor, L. Harms, A. Robinson, R. del Rosario 618 (US 2454897).

Distribución: QRO, S.L.P.

Urochloa jaliscana (F. Santana) Espejo \& López-Ferrari, comb. nov. Basónimo: Brachiaria jaliscana F. Santana, Bol. Inst. Bot. Univ. Guadalajara 1: 183-186, f. 1. 1992 [1993]. TIPO: Jalisco, mpio. Zapopan, predio Las Agujas, $15 \mathrm{~km}$ al W de Zapopan por la brecha a Nextipac, 1600 m, 5.VIII.1985, F. Santana Michel 1375 (holotipo: IBUG; isotipos: ENCB!, MEXU!, MICH!; foto UAMIZ 1820!, WIS, ZEA).

Distribución: JAL.

\section{ORCHIDACEAE}

Juan José Martínez de Lexarza en su Orchidianum Opusculum (1825), dentro de la obra Novorum Vegetabilium Descriptiones realizada en colaboración con Pablo de la Llave, describió 50 especies de orquídeas, la mayoría procedentes de los alrededores de Morelia, Michoacán (antes Valladolid). El material tipo que respaldaba estas descripciones está perdido (Espejo et al., 1993; Stafleu y Cowan, 1979), por lo que la identificación de dichas especies, así como su situación nomenclatural han sido problemáticas. A pesar de lo anterior, el estatus nomenclatural de una buena parte de estos taxa ha sido aclarado (Apéndice 1) e incluso en varios casos se han designado neotipos (Burns-Balogh y Greenwood, 1982; Cribb y Soto-Arenas, 1993; Espejo, 1987; García-Cruz et al., 2000; Greenwood, 1992; Romero y Carnevali, 1999; Sosa, 1992; Soto-Arenas, 1990; Soto-Arenas y Chiang, 1992; Soto-Arenas y Halbinger, 1997).

Entre los nombres que permanecen dudosos se encuentran Epidendrum pastoris y Dendrobium bracteatum. Este último ha sido asignado por Salazar y Soto Arenas al género 
Isochilus, opinión con la cual coincidimos. Dichos autores han anotado diversos ejemplares depositados en los herbarios AMO, ENCB, MEXU, UAMIZ y XAL con la combinación Isochilus bracteatus (Lex.) Salazar y Soto Arenas, sin embargo el binomio nunca ha sido publicado. Por otra parte, el primer nombre corresponde en realidad a una especie del género Prosthechea (Higgins, 1997, 1999). Con el fin de validar ambos nombres para la publicación del Manual de las Orquídeas del Estado de Morelos y el fascículo correspondiente para la Flora del Bajío y de Regiones Adyacentes, formalizamos aquí las combinaciones y designamos los neotipos respectivos. A continuación se reproducen las descripciones originales de Epidendrum pastoris Lex. y Dendrobium bracteatum Lex., seguidas de una descripción actualizada y la cita del neotipo de cada especie.

\section{EPIDENDRUM PASTORIS $\left(^{*}\right)$}

E. Caule repenti, bulbis in seriem ordinatis; foliis elliptico-oblongis; floribus alternis, lamina labelli cordata, acuta; pericarpiis triquetris.

Planta repens, parasitica.- Caulis lignosus, articulatus, nodis membranis imbricatis, laceratis tectis.- Bulbi turbinati, oblongi, paralleli, aequidistantes, perpendiculariter una serie supra caulem ordinati: radiculae spongiosae ad articulos fasciculatae.- Folia bina; raro terna, elliptico oblonga, laeviter striata, subcoriacea, ad extremum bulborum basi scapum amplectentia.- Scapus anceps, nudus, depressus, pauciflorus.- Flores alterni, pedunculati, odorem quasi Vanillae suavissimum emitentes. Bracteolae membranaceae, lineares.Perigonium herbaceo-membranaceum, lutescens, patentissimum, segmentis 5-subaequalibus, lineari-lanceolatis, lineis longitudinalibus purpureo-fuscis, striatis, margine revolutis, acutissimis. Labellum album, ad marcescentiam vi aromatis pallens, cordatum, auriculatum, lineis purpurinis versus basim ad auriculas maculatum, lamella sulcata.- Gynostemum columnare fuscum, ad apicem 3-glandulis luteis coronatum. Anthera opercularis decidua. Pollinis massae 4. Operculum album. Stigma supra canaliculam gynostemi cavatum.- Capsula oblonga, triquetra, angulis acutis. Semina scobiformia.

Habitat supra arbores versus Acuitzium, Undamaeum usque ad Arium in Provincia Michuacanensi, floretque Junio et Julio. (Amatzauhtli species vel Tzacutli, Hern.)

* Diximus in honorem D. Joann. Joss. Pastor Morales, amici dignissimi in scientia Botanica valde periti. $L$.

Prosthechea pastoris (Lex.) Espejo \& López-Ferrari, comb. nov. Basónimo: Epidendrum pastoris Lex., in La Llave \& Lex., Nov. veg. descr. 2: Orchid. opusc. 23-24. 1825. Encyclia pastoris (Lex.) Schltr., Beih. Bot. Centralbl. 36: 473. 1918. TIPO: Michoacán, habitat supra arbores versus Acuitzium [Acuitzio del Canje], Undamaeum [Santiago Undameo] usque ad Arium [Ario de Rosales] in Provincia Michuacanensi, floretque Junio et Julio (probablemente no existe ejemplar). Neotipo (aquí designado): Michoacán, municipio de Morelia, $1 \mathrm{~km}$ antes de Umécuaro, camino Acuitzio - Undameo, 19 32 ' N; 101 ${ }^{\circ} 1^{\prime} \mathrm{W}, 2420$ m. M. A. Soto A. 3071, A. Espejo e I. Ávila sub E. Hágsater 8805 (UAMIZ 50180!; isoneotipos: $\mathrm{AMO}$ !, ENCB!, IEB!). 
Hierbas epífitas, ocasionalmente rupícolas, de hasta $30 \mathrm{~cm}$ de alto. Pseudobulbos fusiformes a ovoides, algo aplanados, de 2 a $8 \mathrm{~cm}$ de largo por 0.3 a $1 \mathrm{~cm}$ de diámetro. Hojas 2, ocasionalmente 3, angostas, elíptico-lanceoladas, obtusas, de 5 a $25 \mathrm{~cm}$ de largo por 0.5 a $1.8 \mathrm{~cm}$ de ancho. Inflorescencia erecta, racemosa, de 8 a $20 \mathrm{~cm}$ de largo; pedúnculo corto, verrugoso, ancipitado, ebracteado; brácteas mucho más pequeñas que las flores, escariosas, triangulares, agudas, de 4 a $6 \mathrm{~mm}$ de largo por ca. $1 \mathrm{~mm}$ de ancho; flores 2 a 4; sépalos y pétalos verdosos con rayas rojizas; labelo blanco con rayas de color rojo vino sobre los lóbulos laterales; ovario ligeramente verrugoso, rollizo, ligeramente ensanchado hacia el ápice, de 2 a $3 \mathrm{~cm}$ de largo por 1 a $1.5 \mathrm{~mm}$ de diámetro; sépalos extendidos, reflejos hacia el ápice, angostamente lanceolados, agudos, de 20 a $25 \mathrm{~mm}$ de largo por 3 a $3.7 \mathrm{~mm}$ de ancho; los laterales ligeramente oblicuos, de 2.2 a $2.7 \mathrm{~cm}$ de largo por 3 a $4.2 \mathrm{~mm}$ de ancho; pétalos extendidos, angostamente lanceolados o elípticos, agudos, de 1.9 a $2.2 \mathrm{~cm}$ de largo por 2 a $2.5 \mathrm{~mm}$ de ancho; labelo unido a la columna en su tercio basal, trilobado, de 1.5 a $2 \mathrm{~cm}$ de largo por 1.3 a $1.8 \mathrm{~cm}$ de ancho; lóbulos aterales extendidos en posición natural, transversalmente elípticos, redondeados, margen entero, separados del lóbulo medio por senos profundos de 1 a $2.5 \mathrm{~mm}$ de ancho; lóbulo medio cordiforme, truncado en la base, agudo, margen entero; disco con una plataforma laminar que se prolonga hasta el ápice del lóbulo medio; columna recta, rolliza, de 8 a $10 \mathrm{~mm}$ de largo, el diente medio oblongo, redondeado, más grande que los laterales y separado de éstos por senos profundos, los laterales oblongos, redondeados; cápsula elipsoide, ligeramente verrugosa, de 3 a $4.5 \mathrm{~cm}$ de largo.

Ejemplares examinados: JALISCO: municipio de Ciudad Guzmán, brecha de El Zapote a El Cacique, 4 km directos al E de Ciudad Guzmán, M. Fuentes O. 404 (AMO, BR); municipio de Ciudad Guzmán, El Floripondio, al suroeste de Cd. Guzmán, R. Soltero Q. 252 (IBUG); municipio de Ciudad Guzmán, El Floripondio, al SW de Cd. Guzmán, Nevado de Colima, R. Soltero Q. 260 (IBUG); municipio de Ciudad Guzmán, cerca de El Floripondio, Nevado de Colima, R. Soltero Q. y Contreras I. 286 (IBUG); municipio de Ciudad Venustiano Carranza, $4 \mathrm{~km}$ al este de El Jazmín, Nevado de Colima, R. Soltero Q. y Contreras I. s. n. (IBUG); municipio de Autlán de Navarro, entre Cacoma y Santa Rosalía, A. Tamayo R. s. n. (IBUG); municipio de Autlán de Navarro, $21 \mathrm{~km}$ al $\mathrm{NO}$ de Autlán, camino a la Iglesia, E. J. Lott y O. Téllez 1147 (MEXU); municipio de La Manzanilla de la Paz, $3 \mathrm{~km}$ al S de La Manzanilla de la Paz, por la brecha Manzanilla-Valle Florido, H. Arreola N. 172 (IBUG); municipio de Tecalitlán, $22 \mathrm{~km}$ SE of highway 110 on a lumber road leaving the highway $12 \mathrm{~km}$ SSW of Tecalitlán and extending to San Isidro, K. Roe y E. Roe 2119 (ENCB); municipio de Tecalitlán, ca. $10 \mathrm{~km}$ al SE de Llanitos, brecha a Plan de Lego, Barranca La Resbalosa, J. Villa C. y J. Chávez L. 240 (CHAPA); municipio de Mazamitla, Mazamitla, J. A. Pérez de la Rosa s. n. (IBUG); municipio de Tuxpan, 26 km al S de Cd. Guzmán, carretera a Colima y $18 \mathrm{~km}$ por brecha a Palos Verdes, J. Gaona P. 383 (AMO); MICHOACÁN: municipio de Charo, aprox. $2 \mathrm{~km}$ al $\mathrm{S}$ después de Las Mesas, en las veredas que suben a la sierra, R. Jiménez M. y J. García-Cruz 2103 (AMO, ENCB, IEB, UAMIZ, XAL); municipio de Charo, aprox. $2.5 \mathrm{~km}$ después de Los Fresnos, sobre la brecha que va a La Escalera, R. Jiménez M. y J. García-Cruz 2122 (AMO, IEB); municipio de Charo, Cerro de la Espadilla, cerca de Las Mesas, S. Martínez 2119 (AMO, ENCB, IEB); municipio de Coeneo, km 55 de la carretera Zacapu-Quiroga, aprox. $10 \mathrm{~km}$ adelante del poblado La 
Constitución, J. García-Cruz 808 (AMO, IEB); 809 (AMO); 812 (AMO); municipio de Coeneo, aprox. $11 \mathrm{~km}$ adelante del poblado La Constitución, en la carretera Zacapu-Quiroga, J. García-Cruz 825 (AMO, IEB, UAMIZ); municipio de Coeneo, en el km 55 de la carretera Zacapu-Quiroga, aprox. $10 \mathrm{~km}$ adelante del poblado La Constitución, R. Jiménez M. 1889 (AMO, IEB); 1890 (AMO); municipio de Coeneo, en el km 55 de la carretera Zacapu-Quiroga, aprox. 10 km adelante del poblado La Constitución, L. Sánchez S. 353 (AMO); 354 (AMO); 355 (AMES, AMO, ENCB, IEB, UAMIZ, XAL); municipio de Erongarícuaro, Tócuaro, H. DíazBarriga 3891 (AMO, ENCB, IEB); municipio de Huaniqueo, N del pedregal grande, $3.5 \mathrm{~km}$ al NE de Jesús María, P. Silva Sáenz 446 (IEB); municipio de Huaniqueo, N del pedregal grande, $2.6 \mathrm{~km}$ al SW de Tendeparacua, P. Silva-Sáenz 1101 (IEB); municipio de Morelia, monte de Arúmbaro, environ de Morelia, H. Galeotti 5214 (W); municipio de Morelia, Morelia, T. Hartweg 396 (W); municipio de Morelia, entre Undameo y Tirio, en las afueras de Morelia, probablemente localidad tipo, G. Morelos s. n. (AMO, IEB); municipio de Morelia, Undameo, G. Morelos sub E. Hágsater 6271 (AMO); municipio de Morelia, Santiago Undameo, G. Morelos sub M. Rodríguez s. n. (AMO); municipio de Morelia, km 231 highway 15, G. E. Pollard 1-190-121 (MEXU); municipio de Morelia, $4 \mathrm{~km}$ al S de San Miguel del Monte, J. Rzedowski 46499a (ENCB); municipio de Morelia, $5 \mathrm{~km}$ al N de Atécuaro, J. Rzedowski 51561 (IEB); municipio de Pátzcuaro, region of Pátzcuaro and SW town Coru, E. A. Aiken 1150 (S); municipio de Salvador Escalante, Copándaro, J. M. Escobedo 990 (AMO, ENCB, IEB), 1967 (AMO, IEB); municipio de Salvador Escalante, Agua Verde, orilla de lago Zirahuén, E. Pérez C. 912 (AMO, ENCB, IEB); municipio de Salvador Escalante, Agua Verde, a la orilla del Lago Zirahuén, J. Rzedowski 40219 (AMO, ENCB, IEB).

Prosthechea pastoris es una especie relativamente abundante en los sitios donde crece. Puede encontrarse como epífita u ocasionalmente rupícola en cañadas húmedas con bosque de encino, bosque de pino-encino y bosque mesófilo entre los 1500 y los 2400 m s.n.m. Se distingue claramente por su labelo blanco con el lóbulo medio cordiforme, apiculado y relativamente grande. Esta vistosa especie fue identificada por muchos años como Encyclia venosa (Lindl.) Schltr.

\section{5o. DENDROBIUM? BRACTEATUM}

D. Caule elongato polyphyllo; foliis distiche vaginantibus linearibus; scapo terminali paucifloro, bracteis concavis subrotundis, magnitudine florum.

Parasitica graminearum habitu.- Caules plurimi elongati, articulati, tenuissimi.- Folia vaginantia disticha, lineari-lanceolata, longissima, nervosa, apice obtusa.- Scapus terminalis brevis, incurvus.- Flores roseii, bracteis magnis concavis alternis cincti. Habitat supra arbores, prope Paracho. Floret Octobri. Obs. Labello differt ab Isochylo graminifolio Humboldtii.

Isochilus bracteatus (Lex.) Salazar \& Soto Arenas, in sched., comb. nov. Basónimo: Dendrobium ? bracteatum Lex., in La Llave \& Lex., Nov. veg. descr. 2: Orchid. opusc. 43. 1825. TIPO: Michoacán, Habitat supra arbores prope Paracho (probablemente no existe 
ejemplar). Neotipo (aquí designado): Michoacán, municipio de Uruapan, pedregal entre Uruapan y Tingambato, $1700 \mathrm{~m}$, bosque de pino y encino sobre basalto, 1.XI.1992, preparado de material cultivado, 18.X.1994, M. A. Soto Arenas et al. 7633 (AMO!; isoneotipos: AMES!, IEB!, K!, UAMIZ!).

Hierbas epífitas, cespitosas, de hasta $50 \mathrm{~cm}$ de alto. Raíces con un grueso velamen y tintes morados. Rizoma repente, cilíndrico. Tallos numerosos, delgados, alargados, de ca. $1.6 \mathrm{~mm}$ de diámetro, multifoliados. Hojas dísticas, glabras, envainantes en la base; las vainas de ca. $2 \mathrm{~cm}$ de largo, las inferiores papilosas; las láminas lineares a linear-lanceoladas, de 3 a $8.5 \mathrm{~cm}$ de largo por ca. 3.5 a $4 \mathrm{~mm}$ de ancho, obtusas y emarginadas en el ápice. Inflorescencia racemosa, secundiflora, terminal, corta, curvada, de 3 a $5 \mathrm{~cm}$ de largo, con 6 a 12 flores densamente dispuestas; brácteas cóncavas, elípticas, tan largas como las flores, de ca. $1 \mathrm{~cm}$ de largo por ca. $6 \mathrm{~mm}$ de ancho; flores rosadas, tubulares, de 8 a 10 $\mathrm{mm}$ de largo; sépalos connados en la base por ca. $2 \mathrm{~mm}$, el dorsal oblongo-elíptico, de ca. $6.5 \mathrm{~mm}$ de largo por ca. $3 \mathrm{~mm}$ de ancho, los laterales oblongo-lanceolados, de ca. 8 $\mathrm{mm}$ de largo por ca. $3 \mathrm{~mm}$ de ancho, con una quilla dorsal muy prominente, con el ápice obtuso; pétalos libres, unguiculados, angostamente elípticos, de ca. $7 \mathrm{~mm}$ de largo por ca. $2 \mathrm{~mm}$ de ancho, con el ápice obtuso; labelo libre, oblongo, sigmoide en la base, con una glándula nectarífera suborbicular, granulosa; columna recta, semirolliza, de ca. $5 \mathrm{~mm}$ de largo por ca. $1.5 \mathrm{~mm}$ de ancho, ventralmente cóncava; ovario subrollizo, con tres costillas inconspicuas, de ca. $7 \mathrm{~mm}$ de largo por ca. $1.5 \mathrm{~mm}$ de diámetro; cápsulas elipsoides, pardas, septicidas, de ca. $9 \mathrm{~mm}$ de largo por ca. $5 \mathrm{~mm}$ de diámetro, con los restos del perianto en el ápice.

Ejemplares examinados: COLIMA: 1.2 miles $S$ of hacienda de San Antonio de Suchitlán, M. W. Chase 82012 (AMO); DURANGO: municipio de Pueblo Nuevo, aproximadamente $1 \mathrm{~km}$ después de La Laguna, por el camino de San Bernardino de Milpillas Chico a San Francisco de Lajas, M. González 2332 (CIIDIR, IEB, UAMIZ); GUERRERO: municipio de Mochitlán, mountains near Mochitlán, O. Nagel y J. González sub E. Oestlund 3316 (AMO); JALISCO: municipio de Autlán, km 26 brecha Autlán - Cacoma, J. A. Pérez de la Rosa 83-B (AMO, IBUG); municipio de Cihuatlán, aproximadamente $1 \mathrm{~km}$ antes del aserradero, rumbo a La Cumbre, A. R. López-Ferrari, A. Espejo, A. Flores y A. Martínez B. 725 (AMO, UAMIZ); municipio de Tecalitlán, $18 \mathrm{~km}$ al sureste de Llanitos, sobre brecha a Plan de Lego, J. Villa y J. Chávez 287 (AMO, CHAPA); municipio de Zapotitlán, 8 km al norte de la hacienda de San Antonio, brecha al Borbollón, J. Villa, S. D. Koch y J. Chávez 847 (AMO, CHAPA); MÉXICO: municipio de Ocuilan, km 12 Ocuilan-Cuernavaca, W. Thurston T-2517 sub E. Hágsater 6086 (AMO, UAMIZ); MICHOACÁN: municipio de Erongarícuaro, Tócuaro, H. Díaz-Barriga 3895 (AMO, IEB, UAMIZ); municipio de Erongarícuaro, pedregal de Tócuaro, J. M. Escobedo 2414 (AMO, IEB); municipio de Uruapan, pedregal de Uruapan, más o menos $5 \mathrm{~km}$ al suroeste de San Andrés Corú, $S$. Zamudio R. y E. Pérez C. 8290 (IEB, UAMIZ); municipio de Zacapu, Malpaís Negro, ca. $7 \mathrm{~km}$ al suroeste de Los Espinos, S. Zamudio y A. Grimaldo 6669 (IEB, UAMIZ); puerto antes Dos Aguas, E. Hágsater 5808 (AMO, UAMIZ); steep mountainsides northwest of Aguililla, ca. 6-7 km south of Aserradero Dos Aguas, R. McVaugh 22694 (AMO, MICH, UAMIZ); MORELOS: municipio de Cuernavaca, al norte de Santa María Ahuacatitlán, 
camino al Tepeite, A. Flores C. 640 (UAMIZ); municipio de Cuernavaca, Mexicapa, carretera Ocuilan-Cuernavaca, I. Luna 470 (MEXU); municipio de Puente de Ixtla, Cerro Frío al sur de Tilzapotla, A. Flores sub A. Espejo 2693 (UAMIZ); NAYARIT: municipio de Tepic, Cuarenteño, E. Hágsater, J. Lamas y M. Bonilla 4485 (AMO, UAMIZ).

Isochilus bracteatus crece como epífita o rupícola en sitios más o menos sombreados entre los 1400 y los 2200 m s.n.m. Es una especie variable en lo referente a los caracteres vegetativos, particularmente en el porte de las plantas y en el largo de las hojas, sin embargo los caracteres florales son bastante constantes.

\section{AGRADECIMIENTOS}

Agradecemos a Jerzy Rzedowski R., Rolando Jiménez M. y Luis Sánchez S. la revisión del manuscrito así como sus valiosas sugerencias y comentarios al mismo. Asimismo deseamos expresar nuestro agradecimiento a los curadores de los diversos herbarios revisados por las facilidades otorgadas para la consulta del material.

\section{LITERATURA CITADA}

Burns-Balogh, P. y E. Greenwood, 1982. Cutsis Balogh, Greenwood and González, a new genus from Mexico. Phytologia 51: 297-298.

Cribb, P. y M. A. Soto-Arenas. 1993. [1994]. The genus Cypripedium in Mexico and Central America. Orquídea (Méx.) 13: 205-214.

Espejo, A. 1987. Neotipificación de Cymbidium vexilliferum La Llave et Lexarza (= Liparis vexillifera) y descripción de Liparis Greenwoodiana. Orquídea (Méx.) 10: 365-384.

Espejo, A., A. R. López-Ferrari y M. Flores. 1993. Neotipificación de Pitcairnia vallisoletana Lex. (Bromeliaceae). Acta Bot. Mex. 23: 53-58.

García-Cruz, J., R. Jiménez M., L. Sánchez S., A. Espejo S. y A. R. López-Ferrari. 2000. Notas sobre el género Habenaria (Orchidaceae) en México. Acta Bot. Mex. 50: 27-38.

Greenwood, E. W. 1992. Govenia liliacea: description and neotypification of a much confused species. Orquídea (Méx.) 12: 155-168.

Higgins, W. E. 1997. A reconsideration of the genus Prosthechea (Orchidaceae). Phytologia 82: 370383.

Higgins, W. E. 1999. The genus Prosthechea: An old name resurrected. Orchids 68: 1114-1125.

La Llave, P. de y J. Lexarza. 1825. Novorum vegetabilium descriptiones 2: Orchidianum opusculum. 43 pp. México.

Romero-González, G. A. y G. Carnevali Fernández-Concha. 1999. Notes on the species of Cyrtopodium (Cyrtopodinae, Orchidaceae) from Florida, Greater Antilles, Mexico, Central and northern South America. Harvard Papers in Botany 4: 327-341.

Sosa, V. 1992. Neotipificación de tres especies del género Bletia (Orchidaceae). Acta Bot. Mex. 18: 71-79.

Soto-Arenas, M. A.1990. Pleurothallis scariosa (Llave \& Lex.) Lindl. In: Hágsater, E. y G. A. Salazar (eds.). Icones Orchidacearum. Fascicle I. Orchids of México. Part 1. plate 93. Asociación Mexicana de Orquideología A. C. México, D. F.

Soto-Arenas, M. A. y F. Chiang. 1992. Maxillaria lexarzana, nuevo nombre para Psittacoglossum atratum. Orquídea (Méx.). 12: 237-243. 
Soto-Arenas, M. A. y F. Halbinger.1997. Laelias of Mexico. Orquídea (Méx.). 15: 1-160.

Stafleu, F. A. y R. S. Cowan. 1979. Taxonomic literature. A selective guide to botanical publications and collections with dates, commentaries and types. 2a. ed. Vol. 2. Utrecht. 991 pp.

Recibido en enero de 2000.

Aceptado en junio de 2000. 


\section{APÉNDICE 1}

\section{ESPECIES DE ORQUÍDEAS PUBLICADAS POR LEXARZA (1825)}

Nombre dado por Lexarza

1. Neottia aurantiaca

2. Neottia cinnabarina *

3. Neottia michuacana

4. Neottia sulphurea

5. Neottia papulosa

6. Neottia micrantha var. a. floribus carneis, labello discolore variegato

7. Cranichis speciosa

8. Cranichis tubularis

9. Satyrium vallisoletanum

10. Orchis entomantha * var. a. floribus herbaceis

11. Ophris macrostachya

12. Cypripedium irapeanum *

13. Cymbidium vexilliferum "vexiliferum" *

14. Maxillaria liliacea *

15. Maxillaria superba

16. Arethusa tigridiaefolia "Aretusa"

17. Bletia punctata *

18. Bletia coccinea *

19. Bletia campanulata * var. a. laciniis perigonii acuminatis: labello concolore miniato

20. Bletia grandiflora

21. Bletia autumnalis *

22. Arpophyllum spicatum

23. Sobralia citrina

24. Epidendrum adenocaulum

25. Epidendrum pastoris

26. Epidendrum adenocarpon *

27. Epidendrum squalidum

28. Epidendrum concolor

29. Epidendrum ligulatum

30. Epidendrum michuacanum

31. Epidendrum anisatum

32. Epidendrum lignosum

33. Pachyphyllum? scandens

34. Psittacoglossum atratum *
Nombre aceptado en la actualidad

Stenorrhynchos aurantiacus (Lex.) Lindl.

Dichromanthus cinnabarinus (Lex.) Garay

Stenorrhynchos michuacanus (Lex.) Lindl.

Stenorrhynchos sulphureus (Lex.) Lindl.

Stenorrhynchos lanceolatus (Aubl.) Rich. ex

Spreng.

Schiedeella llaveana (Lindl.) Schltr. ?

?

Deiregyne rhombilabia Garay ?

Deiregyne pyramidalis (Lindl.) Burns-Bal. ?

Sarcoglottis schaffneri (Rchb. f.) Ames ?

Habenaria entomantha (Lex.) Lindl.

Habenaria sp. nov.

Malaxis macrostachya (Lex.) Kuntze

Cypripedium irapeanum Lex.

Liparis vexillifera (Lex.) Cogn.

Govenia liliacea (Lex.) Lindl.

Govenia superba (Lex.) Lodd.

Bletia purpurata A. Rich. et Galeotti

Bletia punctata Lex.

Bletia coccinea Lex.

Bletia campanulata Lex.

Bletia greenmaniana L. O. Williams

Laelia speciosa (Kunth) Schltr.

Laelia autumnalis (Lex.) Lindl.

Arpophyllum spicatum Lex.

Euchile citrina (Lex.) Withner

Encyclia adenocaula (Lex.) Schltr.

Prosthechea pastoris (Lex.) Espejo et López-

Ferrari

Encyclia adenocarpa (Lex.) Schltr.

Prosthechea linkiana (Klotzsch) W. E. Higgins

Prosthechea concolor (Lex.) W. E. Higgins

?

Prosthechea michuacana (Lex.) W. E. Higgins

Epidendrum anisatum Lex.

Epidendrum lignosum Lex.

Barkeria scandens (Lex.) Dressler et Halb.

Maxillaria lexarzana Soto Arenas et Chiang 
35. Alamania punicea **

36. Cuitlauzina pendula

37. Odontoglossum cervantesii

38. Odontoglossum apterum

39. Odontoglossum maculatum "Odontoglosum"

40. Oncidium tigrinum

var. a. giganteum, scapo ramoso multifloro

var. b. elatum, scapo attenuato, floribus gracillimis

41. Oncidium funereum var. a. bulbis 6-gonis, floribus bicoloribus perig. segmentis inferioribus divergentibus arcuatis var. b. biflorum, labello difformi

42. Oncidium hyalinobulbon "hyalinobulbom"

43. Dendrobium scariosum *

44. Dendrobium retusum

45. Dendrobium quadrifidum

46. Epidendrum? turbinatum

47. Pachyphyllum? uniflorum "Pachiphillum"

48. Epidendrum? (mayzifolium) macrobulbon *

49. Dendrobium? aloideum

50. Dendrobium? bracteatum
Alamania punicea Lex.

Cuitlauzina pendula Lex.

Lemboglossum cervantesii (Lex.) Halb.

Lemboglossum apterum (Lex.) Halb.

Lemboglossum maculatum (Lex.) Halb.

Oncidium tigrinum Lex.

Oncidium tigrinum Lex.

Oncidium tigrinum Lex.

?

?

?

Oncidium hyalinobulbon Lex.

Pleurothallis scariosa (Lex.) Lindl.

Pleurothallis retusa (Lex.) Lindl.

Pleurothallis quadrifida (Lex.) Lindl.

?

Barkeria uniflora (Lex.) Dressler et Halb.

Cyrtopodium macrobulbon (Lex.) G. Romero et Carnevali

?

Isochilus bracteatus (Lex.) Salazar et Soto Arenas

* Nombre para el cual se ha designado neotipo; ${ }^{* *}$ nombre para el cual se conoce material tipo original. 